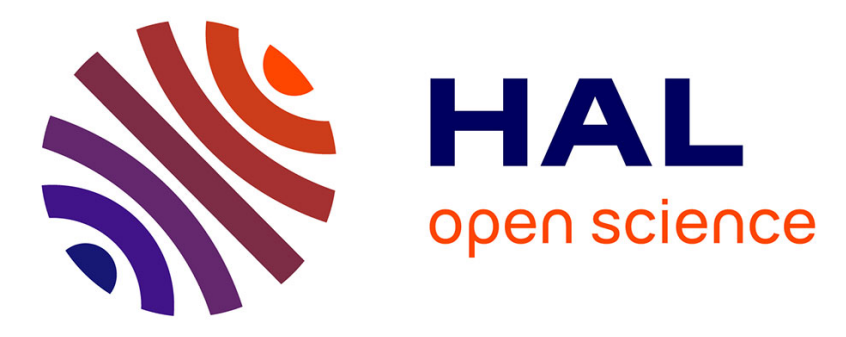

\title{
On Routing for Extending Satellite Service Life in LEO Satellite Networks
}

Mohammed Hussein, Gentian Jakllari, Béatrice Paillassa

\section{To cite this version:}

Mohammed Hussein, Gentian Jakllari, Béatrice Paillassa. On Routing for Extending Satellite Service Life in LEO Satellite Networks. Symposium on Selected Areas in Communications - Satellite \& Space Communication - in IEEE Global Communications Conference (GLOBECOM 2014), Dec 2014, Austin, TX, United States. pp. 2832-2837. hal-01390839

\section{HAL Id: hal-01390839 \\ https://hal.science/hal-01390839}

Submitted on 2 Nov 2016

HAL is a multi-disciplinary open access archive for the deposit and dissemination of scientific research documents, whether they are published or not. The documents may come from teaching and research institutions in France or abroad, or from public or private research centers.
L'archive ouverte pluridisciplinaire HAL, est destinée au dépôt et à la diffusion de documents scientifiques de niveau recherche, publiés ou non, émanant des établissements d'enseignement et de recherche français ou étrangers, des laboratoires publics ou privés. 


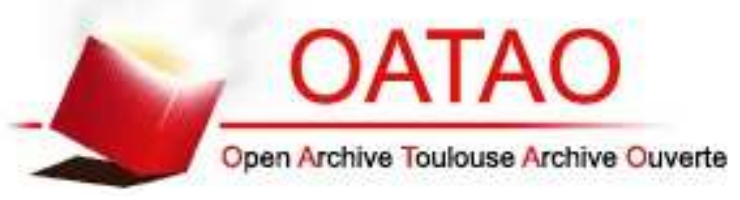

\section{Open Archive TOULOUSE Archive Ouverte (OATAO)}

OATAO is an open access repository that collects the work of Toulouse researchers and makes it freely available over the web where possible.

This is an author-deposited version published in : http://oatao.univ-toulouse.fr/ Eprints ID : 15197

The contribution was presented at GLOBECOM 2014: http://globecom2014.ieee-globecom.org/cfp.html\#.WAde8iSPHWk

To cite this version : Hussein, Mohammed and Jakllari, Gentian and Paillassa, Béatrice On Routing for Extending Satellite Service Life in LEO Satellite Networks. (2014) In: Symposium on Selected Areas in Communications Satellite \& Space Communication - in IEEE Global Communications Conference (GLOBECOM 2014), 8 December 2014 - 12 December 2014 (Austin, TX, United States).

Any correspondence concerning this service should be sent to the repository administrator: staff-oatao@listes-diff.inp-toulouse.fr 


\title{
On Routing for Extending Satellite Service Life in LEO Satellite Networks
}

\author{
Mohammed Hussein, Gentian Jakllari, Beatrice Paillassa \\ IRIT-ENSEEIHT, University of Toulouse, France \\ $\{$ mohammed.hussein, jakllari, beatrice.paillassa\}@enseeiht.fr
}

\begin{abstract}
We address the problem of routing for extending the service life of satellites in Iridium-like LEO constellations. Satellites in LEO constellations can spend over 30\% of their time under the earth's umbra, time during which they are powered by batteries. While the batteries are recharged by solar energy, the depth of discharge they reach during eclipse significantly affects their lifetime - and by extension, the service life of the satellites themselves. For batteries of the type that power Iridium satellites, a $15 \%$ increase to the depth of discharge can practically cut their service lives in half.

We present two new routing metrics - LASER and SLIM

- that try to strike a balance between performance and battery depth of discharge in LEO satellite constellations. Our basic approach is to leverage the deterministic movement of satellites for favoring routing traffic over satellites exposed to the sun as opposed to the eclipsed satellites, thereby decreasing the average battery depth of discharge - all without adversely affecting network performance

Simulations show that LASER and SLIM can reduce the depth of discharge by about $11 \%$ and $16 \%$, respectively, which can lead to as much as $100 \%$ increase in the satellite batteries lifetime. This is accomplished by trading off very little in terms of end-to-end delay.
\end{abstract}

\section{INTRODUCTION}

The past decade has witnessed a growing interest in satellite networks. The satellites architecture is more scalable and provides coverage in harsh environments hard to reach by terrestrial networks. As such, satellite networks are expected be an essential part of the Next-Generation Internet (NGI) [1]. This is particularly the case for LEO satellite constellations that are uniquely positioned to provide the combination of end-to-end delay and data rate required by the bandwidth hungry generation of smartphone applications.

Building and maintaining high performing LEO satellite networks, however, is a daunting challenge - mainly because of two aspects. First, the very environment in which LEO networks operate greatly restricts the processing power and storage capacity of the satellite equipment. Secondly, the high speed movement of LEO satellites results in a highly dynamic, multi-hop topology [2], [3]. As a result, a lot of effort has been put into designing routing protocols for LEO satellite constellations [4], [5], [6], [7].

The emphasis so far has been on performance. The general understanding being that, with LEO satellites being powered by solar energy and batteries - rechargeable by solar energy when under the earth's eclipse - the communication protocols need not be concerned with energy consumption. However, batteries do not last forever. For a constellation like Iridium, satellites spend about $30 \%$ of their time in the earth's umbra [8] - time during which they need to be powered by batteries. Coupled with the fact that it is impractical to replace batteries on satellites it makes the battery lifetime essential to the service time of LEO satellites.

Far and away, the dominant variable affecting the battery lifetime is the depth of discharge (DoD). For nickel hydrogen batteries, the kind of which power the current Iridium constellation satellites, studies have shown that for every $15 \%$ reduction in depth of discharge the battery lifetime almost doubles [9], [10]. Similar behavior is observed with lithium-ion batteries [11], [12], the kind of which will power Iridium NEXT [13].

We present LASER and SLIM, two routing metrics that try to strike a balance between battery lifetime and performance. The basic mechanism employed by both metrics is to: disfavor routing data over satellites that have spent the most time in the earth's umbra - to limit their depth of discharge - while not stretching the paths too much so as to limit the penalty on performance. A key insight in our work is that, unlike other networks, such as sensor networks, where battery lifetime is also essential, the movement of the satellites in a LEO constellation is deterministic. The location of any satellite can be computed and so can if a satellite is eclipsed and for how long [14]. LASER leverages this characteristic to compute the propagation delay and combine it with the satellite battery level acquired via signaling - into a single link metric. SLIM drops all requirements for signaling and instead combines the propagation delay and the time spent in the shadow - both of which can be computed - into a single routing metric.

Simulation results based on publicly available data about the Iridium constellation show that, at least on this practical setting, LASER and SLIM can decrease the depth of discharge by about $11 \%$ and $16 \%$, respectively. This drop in depth of discharge can increase the battery lifetime by as much as $100 \%$ [10], and by extension the LEO satellite service life as well. As the data shows, this improvement is accomplished by trading off very little in terms of endto-end delay.

The remainder of the paper is organized as follows. Section II describes some related work. Section III describes 
how to predict the location of LEO satellite networks. In Section IV we introduce LASER and SLIM. In Section V we present the performance evaluation. Finally, Section VI concludes the paper.

\section{RELATED WORK}

There is a rich literature on routing for LEO satellite constellations (see [4], [5], [6], [7] and references therein) and a thorough review is beyond the scope of this paper. Instead, we present a few representative works in packetswitched solutions to demonstrate the progress in the field while highlighting the need for an approach that takes into account the energy consumption.

In [4], a centralized routing scheme that relies on the Dijkstra's shortest path algorithm to compute the optimal path for any pair of satellites is proposed and evaluated. A centralized scheme can be simple to be deployed and implemented, be that at the ground station or the ingress satellite. However, as with any centralized scheme, it offers low fault-tolerance, and it can impose additional overhead, in the form of larger headers in source routing for example, to deliver the routing information from the centralized node to all satellites. On the other hand, in distributed schemes, routes can be calculated onboard every satellite, based on almost real-time network state information, including link states, Inter Satellite Links (ISL) bandwidth, queue state, traffic distribution etc. Henderson et al [5] proposed a distributed routing algorithm which selects the next hop based on the remaining distance to the destination. Numerical results showed that their solution offered yields good routes, with an average latency degradation of less than 10 msec when compared with the optimal routes. However, in certain cases, such as around the seams and the polar regions the scheme was shown to perform poorly.

The schemes described above use the propagation delay as the main metric. This is to be expected considering the large link lengths in satellite constellations. However, focusing on propagation delay alone can lead to overutilization of and congestion on certain links while leaving other links under-utilized. LAOR, the location assisted ondemand routing protocol for LEO satellite networks [6] tries to remedy this by adapting the AODV [15] protocol to take into account the queueing delay in addition to the propagation delay. However, in an effort to limit the signaling overhead, LOAR limits the scope of RREQs to a specific area between the source and destination. This leads to higher congestion in this particular area resulting in a drop in performance under high loads. T.Taleb. et al [7] claim that a better load balancing algorithm can be achieved by having satellites explicitly notify their neighbors when congestion takes place. Neighboring satellites will respond by decreasing their sending rates and searching for alternative paths. This algorithm is shown to reduce the packet dropping probability, however, it is not protected from signaling congestion due to the very feedback packets. Although congestion-signaling packets are sent only when necessary, they could indeed exacerbate congestion in high load scenarios.

What all the routing protocols described above have in common is their quest for performance. The general understanding has been that, with LEO satellites being powered by solar energy and batteries - rechargeable by solar energy when under the earth's eclipse - the communication protocols need not be concerned with energy consumption. However, satellites in LEO constellations like Iridium can be under the earth's eclipse around $30 \%$ of the time, making batteries essential to their operation. While the batteries are recharged by solar energy, their lifetime is highly affected by the depth of discharge [11], [12]. A routing protocol that, in addition to the performance, is sensitive to the energy consumption of eclipsed nodes, can reduce the depth of discharge and, thus, significantly increase the lifetime of the batteries onboard the satellites. To the best of our knowledge, in this work we present the first effort at building such a routing protocol.

\section{SySTEM MODEL}

In this section, we take advantage of the satellites' and earth's deterministic motions to determine the propagation delays and shadow conditions on a LEO constellation. We use this information in Section IV when designing the new routing metrics.

\section{A. Computing the Propagation Delay over Satellite Links}

As in most publications in the field (e.g. [16], [7], [17]), we consider a single layer Iridium-like polar satellite constellation. The constellation is composed of 6 planes $(\mathrm{N}=6)$, inclined at $86.4^{\circ}$; each plane has 11 satellites $(\mathrm{M}=11)$ that for the need of this study are assumed to be uniformly distributed. Each satellite has four inter satellite links (ISLs): two intra-plane $\left(L_{a}\right)$ ISLs and two interplane $\left(L_{e}\right)$ ISLs, except for the satellites along the counterrotating seam that only have three ISLs. Intra-plane ISLs connect the adjacent satellites in the same plane, while inter-plane ISLs link adjacent satellites across neighboring orbits. The intra-plane ISLs are maintained at all times and their lengths are fixed and can be computed as follows [18]:

$$
L_{a}=\sqrt{2} R \sqrt{1-\cos \left(360^{\circ} \frac{1}{M}\right)}
$$

The inter-plane ISLs are operated only outside the polar region and their lengths vary over time with the satellite movement [18]:

$$
L_{e}=\sqrt{2} R \sqrt{1-\cos \left(360^{\circ} \frac{1}{2 N}\right)} \cos (\text { lat })
$$

Where $\mathrm{R}$ is the radius of the plane and lat stands for the latitude at which the interplane ISL resides.

Using Eq. 1 and Eq. 2, one can compute the propagation delay of a given path. Let $L\left(S_{<n_{1}, m_{1}>}, S_{<n_{q+1}, m_{q+1}>}\right)$ be the length of ISL between satellite $S_{<n_{1}, m_{1}>}$ and satellite $S_{<n_{q+1}, m_{q+1}>}$. The propagation delay of a multihop path can be computed as follows: 


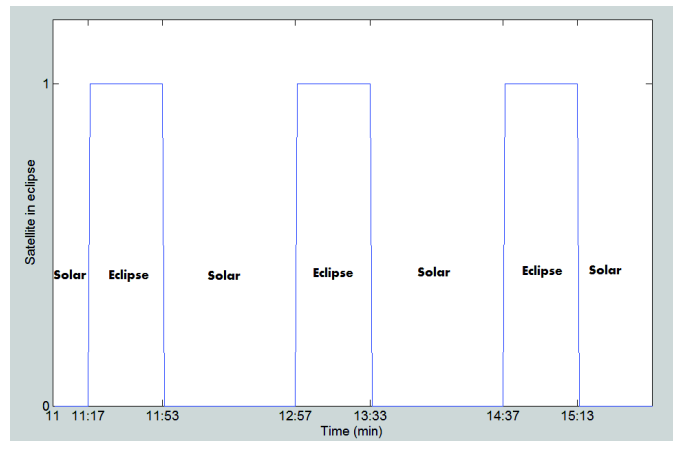

Fig. 1: The analysis is for $\mathbf{2 4}$ hours but for clarity of presentation only a few hours are depicted. This satellite performs a full circle around the earth around 14 times over $24 \mathrm{~h}$, with average cycle duration of around 100 minutes. Out of the 100 minute cycle, around 36 minutes are spent in the earth's umbra.

$$
T_{p}=\frac{\sum_{j=1}^{h(p)} L\left(S_{<n_{1}, m_{1}>}, S_{<n_{q+1}, m_{q+1}>}\right)}{V}
$$

Where $h(p)$ is the number of hops on a specific path and $V$ the speed of light.

\section{B. Computing the LEO Satellite Eclipse Time}

We revisit quickly standard textbook material [19] that can be used to determine, at any given time, whether a particular satellite is under the earth's shadow and for how long.

According to the Kepler model for the circular orbit, we need three quantities to determine the shadow conditions of earth satellites: The orbital size, the orbit inclination $i$, and the right ascension of the ascending node (RAAN), denoted by $\Omega$. The orbit inclination is simply the angle between the orbit plane and the equatorial plane, while RAAN is the angle measured from the vernal equinox along the earth equator to the point at which the satellite ascends from south to north. With this information one can compute the time a particular satellite enters and exits the earth's umbra (shadow) [14].

Figure 1 shows how the computation works for a particular Iridium satellite. The computation is performed using publicly available data about Iridium[8]: altitude 780 kilometers, orbit inclination $86.4^{\circ}$, eccentricity zero, RAAN $235.47^{\circ}$, argument of perigee zero. The experiment begins on September 1, 2013 at 11:00:00 UTC and is carried out for a $24 \mathrm{~h}$ period. For clarity, only a few hours are depicted. We observe that an Iridium satellite performs a full circle around the earth in around 100 mins and spends about 36 minutes in the earth's umbra.

\section{Routing For Extending Satellite Service LIFE}

In this section we present LASER and SLIM, two new routing metrics that try to strike a balance between performance and battery lifetime in LEO satellite constellations.
As mentioned previously, the depth of discharge (DOD) can have a significant impact on the lifetime of batteries deployed onboard satellites. Therefore, our basic approach is to favor routing traffic over satellites exposed to the sun as opposed to the eclipsed satellites functioning on battery energy alone, thereby decreasing the batteries depth of discharge - all without taking a high penalty in performance.

The two proposed metrics present different tradeoffs in terms of signaling overhead and depth of discharge gains. LASER uses signaling for acquiring the level of battery discharge at every satellite and includes that information in the routing metric. SLIM, on the other hand, requires zero signaling and relies solely on the approach described in Section III-B for predicting if a given satellite is in eclipse and for how long.

\section{A. LASER: Loction and loAd SEnsitive Routing}

LASER combines the battery's level of discharge and the propagation delay in creating a new link metric for routing in LEO satellite constellations. Since the motion of satellites is deterministic, the propagation delay can be computed in advance, according to the parameters of selected constellation. The only non-deterministic parameter - the battery level of discharge - will have to be distributed through the network via a flooding mechanism. Once that information is collected, every LEO satellite can compute the LASER value on every link (normalized by the differences of optimal function values [20]) as follows:

$$
\operatorname{laser}_{i, j}(t)=w_{1} \frac{T_{i, j}(t)-T^{\min }}{T^{\max }-T^{\min }}+w_{2} \frac{D_{i, j}(t)-D^{\min }}{D^{\max }-D^{\min }}
$$

Where $T_{i, j}(t)$ is the propagation delay between two satellites, $i, j$, at given time $t$, and $w_{1}$ and $w_{2}$ represent weighting factors that one can tune depending on the application needs ${ }^{1}$. For example, setting $w_{2}=0$ will reduce LASER to a propagation-delay metric. Finally, $D_{i, j}(t)$ is a quantity that depends on the battery levels of the satellites $i$ and $j$ at time $t$ and is computed as follows:

$$
D_{i, j}(t)=\frac{e_{i}}{B_{i}(t)}+\frac{e_{j}}{B_{j}(t)}
$$

In which

- $e=\left\{\begin{array}{l}1, \text { if Satellite is eclipsed by the earth } \\ 0, \text { if Satellite is exposed to the sun }\end{array}\right.$

- $B$ is the residual battery capacity for a given satellite.

As we can see from Eq. 5, the $D_{i, j}$ part of LASER is designed to capture the cost of routing data over satellites whose batteries have higher levels of discharge. Since the goal of LASER is to increase the constellation's service life, it tries to minimize the maximum depth of discharge in the network. To accomplish this, it assigns a higher cost $\left(D_{i, j}\right)$ to batteries with higher levels of discharge while

\footnotetext{
${ }^{1}$ In our simulations we got promising results by setting $w_{1}=w_{2}=$
} 0.5 
obviously assigning zero cost if the solar panels recharging the batteries are exposed to the sun.

Note that, for a given path, its LASER cost is imply the summation of the LASER costs of the links constituting the path.

\section{B. SLIM: SignaL-free routIng for Maximizing satellite service life}

LASER is a first effort at designing a routing metric for minimizing the depth of discharge. However, just like the terrestrial metrics from which it is inspired [21], it requires up-to-date knowledge of battery levels for all satellites possible only with periodic network-wide signaling. This is costly for the eclipsed satellites.

To address this shortcoming of LASER, we introduce SLIM, a metric for SignaL-free routIng for Maximizing satellite lifetime. The key insight behind the SLIM metric is that, unlike many terrestrial networks, the movements of the satellites is deterministic. Thus, at any given time, one can compute for every satellite if it is in eclipse, and if yes, for how long. SLIM uses this information to make less attractive links over satellites that have been in the shadow the most and whose batteries - on average - must have the highest level of discharge:

$$
\operatorname{slim}_{i, j}(t)=w_{1} \frac{T_{i, j}(t)-T^{\min }}{T^{\max }-T^{\min }}+w_{2} \frac{S_{i, j}(t)-S^{\min }}{S^{\max }-S^{\min }}
$$

Where $S(t)$ is the time that a particular satellite has spent in shadow and can be calculated as shown in Section III-B. As with LASER, the SLIM cost of a path is imply the summation of the SLIM costs of the links constituting the path.

LASER vs. SLIM: The energy consumption of an eclipsed satellite does not depend only on how long it has been eclipsed but also the amount of data traffic it has transmitted and received during this period. LASER, who uses the actual battery levels, is more accurate than SLIM. However, LASER requires signaling for acquiring this information, making it heavier than SLIM.

\section{Routing with SLIM and LASER}

There is a rich literature on routing protocols for meshlike topologies in general and LEO constellations in particular. Our goal in this work is not to propose a new routing protocol but rather to propose two routing metrics that can be utilized by current and future routing protocols. Both metrics are additive and thus can be utilized by any routing protocol that uses a least-cost algorithm for computing paths. LASER requires the battery level of all satellites, which can be acquired by including this information in link state updates, for a link state protocol, or the routing discovery packets (RREQ/RREP), for an on-demand protocol like LAOR [6]. SLIM requires no periodic information, except for the initial information to bootstrap the shadowtime computations for every satellite. The periodicity at which SLIM or LASER minimum paths are computed and updated will depend on the particular routing protocol.

\section{Performance Evaluation}

We use the network simulator (ns2.34) [22] as simulation platform and evaluate the performance of LASER and SLIM in terms of battery level of discharge, average endto-end delay, packet delivery ratio and load distribution.

\section{A. Simulation Parameters}

LEO constellation parameters: Our performance analysis is based on the Iridium constellation [8]. There are 6 orbital planes with 11 satellites each and inter-plane separation of $31.6^{\circ}$; each satellite is assumed to have four ISLs with two intra-orbits ISLs and two inter-orbits ISLs. The bandwidth of Uplink-Downlink (UDL) and ISL links are $1.5 \mathrm{Mbps}$ and $10 \mathrm{Mbps}$, respectively. We do not consider the seams where two ISLs are switched off due to the motion in opposite direction. Satellite orbits are $780 \mathrm{~km}$ in altitude with an orbit inclination angle of $86.4^{\circ}$ and the minimum elevation angle of ground stations is $8.2^{\circ}$. Mission started Dec 1, 2013 at 11:00:00 UTC.

Battery parameters: To make the simulations as realistic as possible, we use publicly available data for the Iridium satellites. Specifically, the battery capacity is set to 117 Kjoule, transmission power to 7 watt, reception power to 3 watt and the nominal operation power to 4 watt.

Routing protocols: While the SLIM and LASER metrics can be implemented over any routing protocol, we implemented them over Dijkstra's Shortest Path (DSP) for simplicity. For SLIM we implement a standard link state update protocol [23] for collecting the battery levels of all satellites.

Basis for comparison: We compare SLIM and LASER to pure DSP, for two reasons. First, DSP remains one of the most popular routing methods for LEO satellite networks [24]. Second, comparing to a protocol that ignores the battery lifetime helps quantify the potential for improvement and tradeoffs involved in switching to protocols that do take the battery lifetime into account.

\section{B. Experiment 1: Battery Depth of Discharge}

We start with an experiment for evaluating LASER and SLIM in terms of battery depth of discharge. For this, 100 terminals are distributed over six continents according to the distribution used in [25] and a CBR traffic generator transmitting at $1.5 \mathrm{Mbps}$ is attached to each one of them. The average packet size is set to 210 Byte. Unlike DSP and SLIM, LASER requires extra control packets for acquiring the battery levels so to make the comparison fair we have associated an energy cost to every control packet.

Figure 2(a) compares the CDFs of the battery levels at the end of the eclipse period for all satellites when using LASER, SLIM and pure DSP. The data shows that LASER and SLIM reduce the level of discharge by as much as $11 \%$ and $16 \%$, respectively, when compared to DSP. Considering 


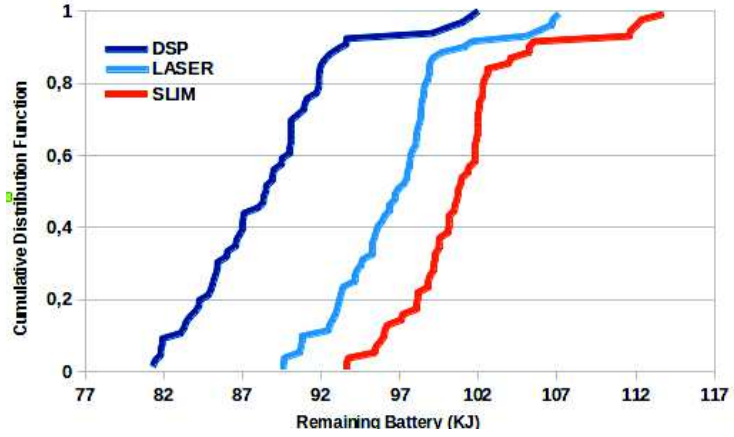

(a) The CDF of battery levels for all the 66 satellites. The $x$ - axis represents the battery level just before exiting eclipse.

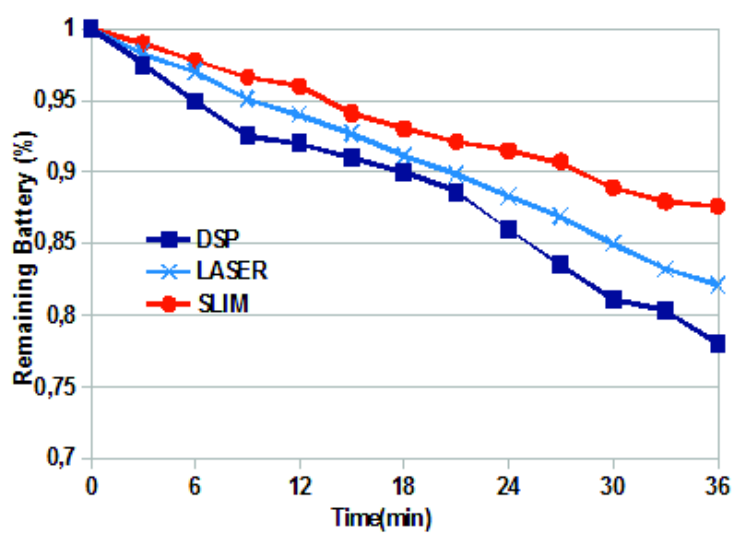

(b) Battery level for one satellite as it goes through eclipse. Out of the 100 minute cycle, around 36 minutes are spent in the earth's umbra

Fig. 2: LASER and SLIM reduce the depth of discharge (DOD) by as much as $11 \%$ and $16 \%$ (median $10 \%$ and $14 \%$ ), respectively, over a metric that does not take battery discharge into account. Considering that reducing a nickel hydrogen battery's DOD by $15 \%$ almost doubles its lifecycle, using SLIM can significantly extend the LEO satellites service life. The data also shows that SLIM's "lightness" outperforms LASER's accuracy.

the effect the depth of discharge has on the battery lifetime [10] [12], a $16 \%$ reduction in the depth of discharge can double the satellite service life in LEO constellations. For further clarity, in Figure 2(a) we zoom into an arbitrarily satellite as it goes through the eclipse period. Once the satellite enters the eclipse its battery level starts dropping. However, the drop is less pronounced for LASER and SLIM.

Finally, the data shows that SLIM's "lightness" outperforms LASER's accuracy.

\section{Experiment 2: The Service Life-Performance Tradeoff}

In the second part of the evaluation we turn our attention to the tradeoff between performance and satellite service life. Towards this, we perform a second experiment with settings like the first except that we vary the traffic input rate from 0.5 to $1.5 \mathrm{Mbps}$ to emulate different levels of

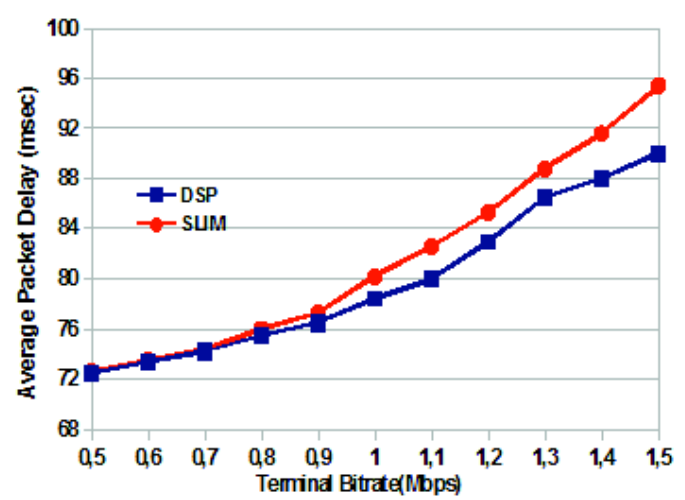

Fig. 3: As expected, with SLIM there is a slight increase in end-to-end delay. The bigger gap for higher load is in part due to the fact that, as shown in Fig. 4(b), for these values the network is congested and SLIM delivers more packets than DSP.

traffic load. We measure the end-to-end delay, the load distribution index as well as the packet delivery ratio for SLIM and DSP. We omit LASER for this part of the evaluation since SLIM was shown to outperform it.

Figure 3 shows the results for the end-to-end delay. As expected, the SLIM's improvement to the battery depth of discharge does not come entirely free - a slight increase in the end-to-end delay over the DSP is observed. Note, however, that the biggest increase is observed for the high loads and is due to the fact that SLIM simply delivers more packets (as shown in Figure 4(b) and elaborated below). Furthermore, the data indicates that setting $w_{1}=w_{2}=$ 0.5 for SLIM (see Eq. 6) strikes a good balance between performance and service life.

To get a better understanding of the network behavior with SLIM, in Figure 4, we depict the packet delivery ratio and the load distribution index, $f$ [7]:

$$
f=\frac{\left(\sum_{i=1}^{n} x_{i}\right)^{2}}{n \sum_{i=1}^{n} x_{i}^{2}}
$$

Where $n$ is the number of satellites and $x_{i}$ denotes the number of packets that traversed the $i^{\text {th }}$ satellite.

Figure 4(a) shows that using SLIM leads to a much better load distribution over the LEO constellation when compared to DSP. This is to be excepted since SLIM adapts to the position and battery level of the satellites while DSP always uses the shortest path to the destination. At high loads this can lead to the shortest paths becoming congested which - as the data in Figure 4(b) shows - explains why with SLIM the packet delivery ratio is better for high loads.

\section{CONCLUSION}

In this work, we proposed two routing metrics, LASER and SLIM that try to strike a balance between extending the LEO satellites service life and performance. The key intuition underlying LASER and SLIM is that eclipsed satellites - powered by batteries - should be less favored 


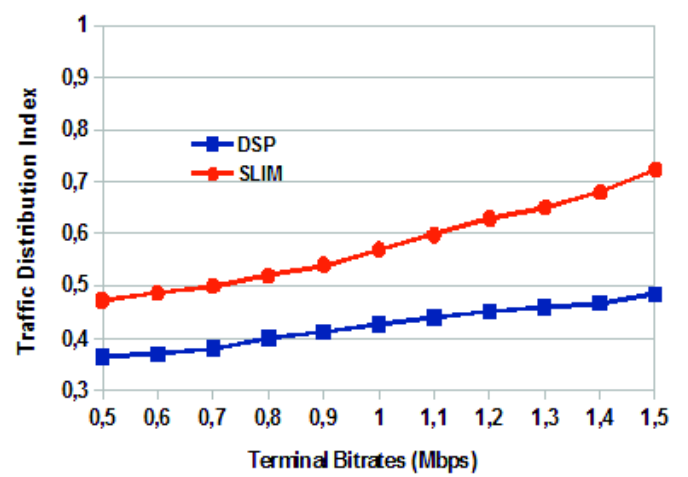

(a) Load Distribution Index for different terminal bitrates

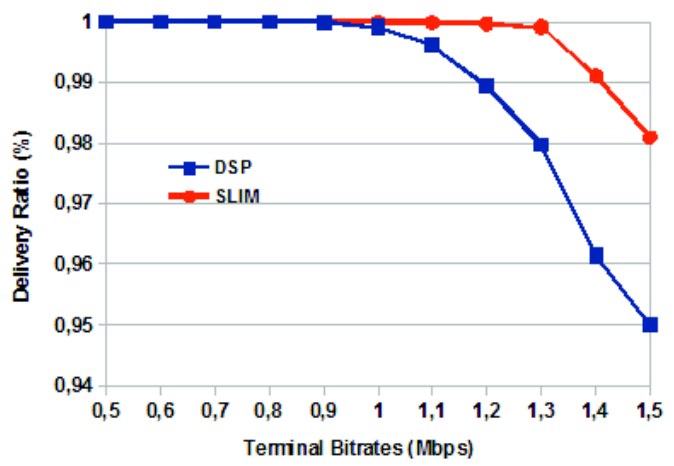

(b) Packet Delivery Ratios for different terminal bitrates

Fig. 4: SLIM adapts to the location and battery level of satellites while DSP always selects the shortest paths. As a result, using SLIM leads to better load distribution, and better delivery ratio at high loads.

for routing data traffic when compared to satellites exposed to the sun. Our simulation analysis showed that LASER and SLIM could lead to significant improvement in the battery depth of discharge and by extension to increased satellite service life. This was accomplished by trading off very little in terms of end-to-end delay.

As future work, we intend to extend LASER and SLIM to take into account the link error rate and evaluate their performance across a more rich set of parameters and on a more realistic setting.

\section{REFERENCES}

[1] N. Courville, H. Bischl, E. Lutz, A. Svigelj, E. L. De, P. M. L. Chan, R. Road, and R. Asorey-cacheda, "Hybrid satellite / terrestrial networks : State of the art and future perspectives," Satellite/Terrestrial Interworking (IWSTI) Workshop, 2007.

[2] S. Karapantazis, E. Papapetrou, and F.-N. Pavlidou, "On-Demand Routing in LEO Satellite Systems," 2007 IEEE International Conference on Communications, pp. 26-31, Jun. 2007.

[3] Q. Yang, D. I. Laurenson, and J. A. Barria, "On the use of LEO satellite constellation for active network management in power distribution networks," Smart Grid, IEEE Transactions on, vol. 3, no. 3, pp. 1371-1381, 2012.
[4] M. Mohorcic, M. Werner, A. Svigelj, and G. Kandus, "Adaptive routing for packet-oriented intersatellite link networks: performance in various traffic scenarios," Wireless Communications, IEEE Transactions on, vol. 1, no. 4, pp. 808-818, Oct 2002.

[5] T. R. Henderson, M. Park, and R. H. Katz, "On distributed and geographic-based packet routing for LEO satellite networks," IEEE Globecom, pp. 1119-1123, 2000.

[6] E. Papapetrou, S. Karapantazis, and F.-N. Pavlidou, "Distributed on-demand routing for LEO satellite systems," Computer Networks, vol. 51, no. 15, pp. 4356-4376, Oct. 2007. [Online]. Available: http://linkinghub.elsevier.com/retrieve/pii/S1389128607001582

[7] T. Taleb, D. Mashimo, A. Jamalipour, N. Kato, and Y. Nemoto, "Explicit Load Balancing Technique for NGEO Satellite IP Networks With On-Board Processing Capabilities," Networking, IEEE/ACM Transactions on, vol. 17, no. 1, pp. 281-293, Feb 2009.

[8] S. Q. Kidder and T. H. V. Haar, "A satellite constellation to observe the spectral radiance shell of earth," in Proceedings of 13th Conference on Satellite Meteorology and Oceanography, Norfolk, Virginia, USA, 2004.

[9] L. H. Thaller and T. P. Barrera, "Modeling performance degradation in nickel hydrogen cells," DTIC Document, Tech. Rep., 1991.

[10] A. Dutta and Y. Yemini, "Power management of LEOs under bursty broadband traffic," 17th AIAA International Communications Satellite Systems Conference and Exhibit, pp. 1-11, Feb. 1998. [Online]. Available: http://arc.aiaa.org/doi/abs/10.2514/6.1998-1317

[11] G. Ning and B. N. Popov, "Cycle life modeling of lithiumion batteries," Journal of The Electrochemical Society, vol. 151, no. 10, pp. A1584-A1591, 2004. [Online]. Available: http://jes.ecsdl.org/content/151/10/A1584.abstract

[12] J.-W. Lee, Y. K. Anguchamy, and B. N. Popov, "Simulation of chargedischarge cycling of lithium-ion batteries under low-earthorbit conditions," Journal of Power Sources, vol. 162, pp. 13951400, 2006.

[13] "Iridium next: A global effort to launch the future of global communications," Iridium Press release, August 2013. [Online]. Available: http://www.iridium360.com/2013/04/19/iridium-next-aglobal-effort-to-launch-the-future-of-global-communications/

[14] C. R. O. Longo and S. L. Rickman, "Method for the Calculation of Spacecraft Umbra and Penumbra Shadow Terminator Points," NASA Technical Paper 3547, 1995.

[15] C. Perkins, E. Belding-Royer, and S. Das, "Ad hoc on-demand distance vector (aodv) routing," United States, 2003.

[16] Y. Rao and R.-c. Wang, "Agent-based load balancing routing for LEO satellite networks," Computer Networks, vol. 54, no. 17 , pp. 3187-3195, Dec. 2010. [Online]. Available: http://linkinghub.elsevier.com/retrieve/pii/S1389128610002215

[17] K. Maine, C. Devieux, and P. Swan, "Overview of IRIDIUM satellite network," Proceedings of WESCON'95, p. 483. [Online]. Available: http://ieeexplore.ieee.org/lpdocs/epic03/wrapper.htm?arnumber=485428

[18] E. Ekici, I. F. Akyildiz, and M. D. Bender, "A distributed routing algorithm for datagram traffic in LEO satellite networks," Networking, IEEE/ACM Transactions on, vol. 9, no. 2, pp. 137-147, 2001.

[19] J. R. Wertz, Mission Geometry: Orbit and Constellation Design and Management. El Segundo, California: Microcosm, Inc., 2001.

[20] O. Grodzevich and O. Romanko, "Normalization and other topics in multi-objective optimization," In Proceedings of the Fields MITACS Industrial Problems Workshop, Toronto, 2006.

[21] A. Misra and S. Banerjee, "MRPC: Maximizing network lifetime for reliable routing in wireless environments," in Wireless Communications and Networking Conference, 2002. WCNC2002. 2002 IEEE, vol. 2. IEEE, 2002, pp. 800-806.

[22] “The network simulator - ns2." [Online]. Available: Http://isi.edu/nsnam/ns/

[23] "Optimized link state routing protocol (olsr)," United States, 2003.

[24] H. Nishiyama, Y. Tada, N. Kato, N. Yoshimura, M. Toyoshima, and N. Kadowaki, "Toward Optimized Traffic Distribution for Efficient Network Capacity Utilization in Two-Layered Satellite Networks," IEEE Transactions on Vchicular Technology, vol. 62, pp. 1303-1313, 2012.

[25] A. Svigelj, M. Mohorcic, G. Kandus, A. Kos, M. Pustisek, and J. Bester, "Routing in ISL networks considering empirical IP traffic," Selected Areas in Communications, IEEE Journal on, vol. 22, no. 2, pp. 261-272, 2004. 